\title{
INFLUÊNCIA DA CORREÇÃO DA IDADE NA DETECÇÃO DE RISCOS NO DESENVOLVIMENTO MOTOR DE PREMATUROS
}

\section{Natiele Camponogara Righi}

Fisioterapeuta. Universidade Federal de Santa Maria (UFSM), Brasil.

E-mail: natirighi@gmail.com

\section{Fabiane Kurtz Martins}

Fisioterapeuta. Universidade Federal de Santa Maria (UFSM), Brasil.

\section{Leticia Hermes}

Mestranda no Mestrado Profissional em Ciências da Saúde pela Universidade Federal de Santa Maria (UFSM), Brasil.

\section{Kauene Marques da Rosa}

Acadêmica do Curso de Fisioterapia Universidade Federal de Santa Maria (UFSM), Brasil.

\section{Thais Helena Oliveira Böck}

Acadêmica do Curso de Fisioterapia Universidade Federal de Santa Maria (UFSM), Brasil.

\section{Claudia Morais Trevisan}

Docente Associada do Departamento de Fisioterapia e Reabilitação da Universidade Federal de Santa Maria (UFSM), Brasil.
RESUMO: Avaliar a influência da correção da idade na detecção de riscos no desenvolvimento motor de prematuros. Trata-se de um estudo observacional retrospectivo, com 59 prematuros, atendidos no Ambulatório de Seguimento de Prematuros de um hospital-escola de uma cidade da região Central do Rio Grande do Sul, nos anos de 2014 e 2015. As crianças foram avaliadas a partir da Albert Infant Motor Scale aos quatro e oito meses. A normalidade dos dados foi verificada pelo teste de Shapiro-Wilk e a comparação dos grupos foi realizada a partir do Teste de Wilcoxon. Utilizando a idade corrigida, 39\% dos prematuros avaliados aos quatro meses apresentaram alto risco para atraso do desenvolvimento motor, assim como $33 \%$ dos prematuros avaliados aos oito meses. Este resultado foi mais acentuado quando a idade cronológica foi utilizada $(p=0,000)$. A correção da idade gestacional se mostrou como a melhor forma de avaliar o desempenho real dos bebês nascidos pré-termo, sem superestimar riscos que não se configuram de fato em problemas no desenvolvimento das crianças.

PALAVRAS-CHAVE: Prematuro; Desenvolvimento infantil; Avaliação.

\section{INFLUENCE OF AGE ALTERATION IN RISK DETECTION IN THE MOTOR DEVELOPMENT OF PREMATURE CHILDREN}

\begin{abstract}
Current paper assesses the influence of age correction when risks in the motor development of premature children are detected. A retrospective and observational study has been conducted with 59 premature children attended to at the Premature Children Clinic of a school hospital in the central region of the state of Rio Grande do Sul, Brazil, between 2014 and 2015. The children were evaluated by the Albert Infant Motor Scale, at ages four and eight months. Data normality was verified by the Shapiro-Wilke's test and group comparison was undertaken by Wilcoxon's Test. 39\% of the premature children evaluated on month four revealed high risk levels for delay in motor development; similar results were obtained for 33\% of premature children evaluated on month eight. Results were more significant when chronological age was employed $(p=0.000)$. The correction of gestation age proved to be the best way in evaluating the true performance of pre-term children, without highly estimating risks which do not actually emerge into problems in children's development.
\end{abstract}

KEY WORDS: Premature children; Child development; Evaluation. 


\section{INTRODUÇão}

Estima-se que, a cada ano, ocorra cerca de 15 milhões de nascimentos prematuros, definido pela Organização Mundial da Saúde como aquele que acontece antes da $37^{\mathrm{a}}$ semana gestacional (WHO, 2015). No Brasil, a média fica em torno de 12,3\% (LAJOS, 2014). Tal condição é considerada prejudicial ao recém-nascido, pelo maior risco de ocorrência de complicações clínicas decorrentes da imaturidade dos sistemas orgânicos do bebê (FORMIGA; LINHARES, 2011).

Os recém-nascidos prematuros (RNPT) apresentam maiores riscos de atraso no desenvolvimento motor, pois são privados da experiência de estar em um ambiente com pequeno espaço (intrauterino), que o fará diminuir sua movimentação mais ampla e da sobrevida desses, o que ocorre principalmente pelos avanços tecnológicos e esforços colaborativos para começar a desenvolver um tônus flexor (BRASIL, 2011). Apesar do aumento progressivo de partos prematuros, é evidenciado aumento na taxa de prematuros sobreviventes da equipe de saúde (MENETRIER; ALMEIDA, 2016)

Por esse motivo, o acompanhamento do RNPT, em um seguimento ambulatorial, é fundamental, sendo possível dar atenção especial ao desenvolvimento nos primeiros anos de vida, período de grandes modificações e aquisições de habilidades, quando os desvios da normalidade, os distúrbios e atrasos manifestam-se e precisam ser detectados e assim identificadas as crianças que necessitam intervenção precoce (RYDZ et al., 2005)

$\mathrm{Na}$ avaliação do desenvolvimento do RNPT pode ser utilizada a idade corrigida (ICo), que traduz o ajuste da idade cronológica (IC) em função do grau de prematuridade ao subtrair da IC as semanas que faltaram para a idade gestacional atingir 40 semanas (FORMIGA; LINHARES, 2011). Ao corrigir a idade gestacional nos primeiros dois anos de idade, espera-se que as sequências do desenvolvimento dos RNPT tornem-se semelhantes às apresentadas por crianças nascidas a termo, a fim de evitar um diagnóstico falso-negativo de atraso motor.

Apesar disso, a utilização da correção da idade gestacional do RNPT ainda apresenta controvérsias na literatura. No estudo de Raniero et al. (2010), ao comparar lactentes pré-termo e a termo, foi concluído que não é necessária a correção do grau de prematuridade nos primeiros quatro meses e os estudos de Restiffe e Guerpelli (2006) e Saccani e Valentine (2010), os quais apontaram que a avaliação do desenvolvimento utilizando a idade cronológica pode superestimar riscos ou problemas no primeiro ano de idade, devendo esta ser corrigida.

Nesta perspectiva, o objetivo do estudo foi avaliar a influência da correção da idade na detecção de riscos no desenvolvimento motor de prematuros.

\section{METODOLOGIA}

Estudo observacional retrospectivo, com 59 prematuros, atendidos no Ambulatório de Seguimento de Prematuros de um hospital-escola de uma cidade da região Central do Rio Grande do Sul, nos anos de 2014 e 2015. Os prematuros foram divididos em dois grupos: quatro meses (38 casos) e oito meses ( 21 casos) de ICo.

As crianças foram avaliadas a partir da Albert Infant Motor Scale (AIMS), que se baseia na observação de recém-nascidos até 18 meses de idade, em quatro posturas quanto ao tempo de permanência, atitude antigravitacional e mudança de posturas. O escore obtido pela criança em cada postura (prono, supino, sentado e em pé) é somado para pontuação final. Esta é lançada no gráfico para a classificação do percentil de acordo com a idade do bebê. Na interpretação dos resultados, o desenvolvimento é classificado como normal entre 25\% e $90 \%$ da curva percentílica; suspeito, abaixo de $10 \%$ da curva percentílica após o sexto mês; e alto risco, 10\% e $5 \%$ da curva percentílica aos quatro meses e $5 \%$ da curva percentílica aos oito meses.

A normalidade dos dados foi verificada pelo teste de Shapiro-Wilk e a comparação dos grupos foi realizada a partir do teste de Wilcoxon. As análises foram realizadas utilizando o programa Statistical Package for the Social Sciences for Windows, versão 17.0 para Windows, com nível de significância de $5 \%(\mathrm{p}<0,05)$ para todos os testes.

O estudo foi aprovado pelo Comitê de Ética em Pesquisa sob parecer número 1.218.985 e o termo de consentimento livre e esclarecido foi assinado pelos responsáveis. 


\section{RESULTADOS}

Foram estudados 59 recém-nascidos pré-termo, com peso de nascimento médio 1476,69 \pm 595,14 gramas, idade gestacional (IG) média 30,67 \pm 2,86 semanas, sendo $50,8 \%$ do sexo masculino e $49,1 \%$ do sexo feminino (Tabela 1).

Tabela 1. Características da população estudada. Santa Maria/ RS, 2017

\begin{tabular}{cc}
\hline Peso ao nascimento (gramas) & $1476,69 \pm 595,14$ \\
Idade gestacional (semanas) & $30,67 \pm 2,86$ \\
\hline Sexo n (\%) & $29(49,1 \%)$ \\
\hline Feminino & $30(50,8 \%)$ \\
Masculino & \\
\hline
\end{tabular}

De toda a amostra, 37\% dos prematuros apresentaram alto risco para atraso do desenvolvimento motor. Utilizando a ICo, 39\% dos prematuros avaliados aos quatro meses apresentaram alto risco para atraso do desenvolvimento motor, assim como 33\% dos prematuros avaliados aos oito meses. Este resultado foi mais acentuado quando a IC foi utilizada (Figura 1), apresentando diferenças estatisticamente significantes (Tabela 2).

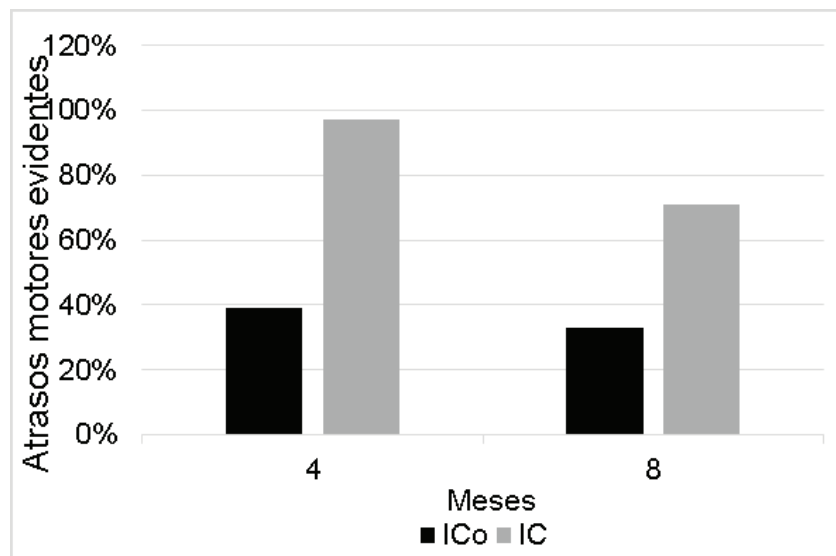

Figura 1. Porcentagem de crianças com atrasos motores evidentes. Santa Maria/RS, 2017

Fonte: Autor
Tabela 2. Crianças com atrasos motores evidentes. Santa Maria/RS, 2017

\begin{tabular}{cccc}
\hline Variáveis & $\begin{array}{c}4 \text { meses } \\
\text { Mediana }\end{array}$ & $\begin{array}{c}\mathbf{8} \text { meses } \\
\text { Mediana }\end{array}$ & p-valor* \\
\hline IC & 5 & 25 & $0,000^{*}$ \\
ICo & 5 & 25 & $0,000^{*}$ \\
\hline
\end{tabular}

IC, Idade cronológica; ICo, Idade corrigida

\section{DISCUSSÃO}

Os resultados deste estudo, que objetivou avaliar o desenvolvimento motor de recém-nascidos prematuros (RNPT), pela Albert Infant Motor Scale, comparando as idades cronológica (IC) e corrigida (ICo), aos quatro e oito meses, mostraram diferença na porcentagem de bebês com alto risco para atraso do desenvolvimento motor. Este risco aumentou quando se considerou o desempenho dos RNPT na IC, em comparação a ICo em comparação a ICo. Estes resultados concordam com estudos anteriores, como o de Saccani e Valentini (2010), que detectaram diferença significativa no desempenho motor das crianças ao comparar os resultados sobre a ICo e a IC, demonstrando a importância de se considerar a correção da idade gestacional ao avaliar o desempenho de crianças até 18 meses de idade.

Volpi et al. (2010) observaram que a correção da idadedeveserfeitaatéaaquisiçãoda marchaindependente, Ao avaliar 155 prematuros em um estudo que objetivou determinar as idades cronológica e corrigida de aquisição das habilidades motoras até a marcha independente em prematuros de muito baixo peso e avaliar até quando é necessário o uso da idade corrigida. Além disso, Restiffe e Gherpelli (2006), ao verificar a necessidade de corrigir a IC de acordo com o grau de prematuridade, na avaliação do desenvolvimento motor grosseiro em recém-nascidos pré-termo, concluíram que a ICo deve ser utilizada na avaliação do desenvolvimento do motor de RNPT durante o primeiro ano de vida.

Assim como no estudo anterior, em sua revisão comprobatória, D'Agostino (2010) concluiu que o uso de ajuste do grau de prematuridade foi apoiado para a avaliação dos prematuros por um período mínimo de 12 meses de ICo, pois este melhora a capacidade 
de reconhecer de forma precisa atrasos genuínos em oposição a atrasos perceptíveis relacionados com a idade gestacional de uma criança no momento do nascimento. Os resultados do presente estudo concordam com a literatura, evidenciando a importância de considerar a correção da idade gestacional ao avaliar o desempenho motor de prematuros, para não acarretar em falso diagnóstico de atraso no desenvolvimento motor.

Assim como a função motora grossa, estudos indicam que a correção da prematuridade favorece 0 desempenho motor fino das crianças. Piper et al. (1989) e Mancini et al. (2000), ao avaliarem a motricidade fina de crianças prematuras, considerando a IC e a ICo, encontraram diferença entre as análises. No entanto, da mesma maneira que Ouden et al. (1991) apontam que a correção da idade parece superestimar o desempenho motor à medida que a criança se aproxima dos 24 meses de idade, alegando que esta deve ser aplicada no primeiro ano de vida, não sendo necessária no segundo ano.

Além do desenvolvimento motor, a literatura sugere a hipótese de que a idade gestacional no momento do nascimento exerce influência na linguagem, aprendizagem, atenção e no comportamento visual da criança (RÍOS-FLÓRE; NOVOA-SUAZA; HERNÁNDEZHENAO, 2017; MACHADO et al., 2017). Albuquerque et al. (2009), ao avaliar o comportamento visuomotor de prematuros no primeiro mês de vida, levando em consideração as idades cronológica e corrigida, observaram que a maioria dos recém-nascidos prematuros apresentaram maior contato visual, sorriso como uma resposta social, acompanhamento visual horizontal e vertical e aumento da mobilidade dos membros superiores em ver o objeto, foram os que apresentaram a ICo. O estudo revela a importância de se levar em conta a ICo na medição dos parâmetros envolvidos no desenvolvimento do comportamento visuomotor.

De toda a amostra, 37\% dos prematuros apresentaram alto risco para atraso do desenvolvimento motor. Tal situação requer assistência especializada e multidisciplinar, sendo necessário o acompanhamento periódico dessas crianças, como acontece nos ambulatórios de seguimento. Nele, as crianças são avaliadas de forma integral, com vista à detecção e intervenção precoces das intercorrências com atenção especial no acompanhamento do crescimento, nutrição, funções visuais, auditivas, neuromotoras, cognitivas, imunização e cuidado domiciliar (TRONCHIN; TSUNECHIRO, 2007).

\section{CONCLUSÃO}

A correção da idade gestacional se mostrou como a melhor forma de avaliar o desempenho real dos bebês nascidos pré-termo, sem superestimar riscos que não se configuram, de fato, em problemas no desenvolvimento das crianças.

\section{REFERÊNCIAS}

ALBUQUERQUE, R. C. et al. Visuomotor behaviour of preterm infants in the first month of life. A comparison between the chronological and corrected ages. Revista de Neurologia, v. 48, n. 1, p. 13-16, 2009.

BRASIL. Ministério da Saúde. Atenção humanizada ao recém-nascido de baixo peso - Método Canguru: Manual técnico. 2. ed. Brasília, DF: [s.n.], 2011.

D'AGOSTINO, J. A. An Evidentiary Review Regarding the Use of Chronological and Adjusted Age in the Assessment of Preterm Infants. Journal for Specialists in Pediatric Nursing, v. 15, n. 1, p. 26-32, 2010.

FORMIGA, C. K. M. R.; LINHARES, M. B. M. Follow-up do desenvolvimento do bebê de risco. In: SARMENTO, G.J.V. Fisioterapia respiratória em pediatria e neonatologia. 2. ed. Barueri: Manole, 2011. p. 556-573.

LAJOS, G.J. Estudo multicêntrico de investigação em prematuridade no Brasil: implementação, correlação interclasse e fatores associados à prematuridade espontânea. Campinas, SP: [S.n.], 2014.

MACHADO, A. C. C. P. et al. Processamento sensorial no período da infância em crianças nascidas pré-termo: revisão sistemática. Revista Paulista de Pediatria, v. 35 , n. 1, p. 92-101, 2017. 
MANCINI, M. C. et al. Comparação das habilidades motoras de crianças prematuras e crianças nascidas a termo. Fisioterapia e Pesquisa, v. 7, n. 1/2, p. 25-31, 2000.

MENETRIER, J. V.; ALMEIDA, G. Perfil epidemiológico de gestantes de alto risco com parto prematuro em um hospital de referência. Revista Saúde e Pesquisa, v. 9, n. 3, p. 433-441, 2016.

OUDEN, L. D. et al. Is it correct to correct? Developmental milestones in 555 "normal" preterm infants compared with term infants. Journal of Pediatrics, v. 118, p. 399404, 1991.

PIPER, M. C. et al. Gross and fine motor development of preterm infants at eight and 12 months of age. Developmental Medicine \& Child Neurology, v. 31, p. 591-597, 1989.

RANIERO, E. P.; TUDELLA, E.; MATTOS, R. S. Pattern and rate of motor skill acquisition among preterm infants during the first four months corrected age. Revista Brasileira de Fisioterapia. v. 14, n. 5, p. 396-403, 2010.

RESTIFFE, A. P.; GHERPELLI, J. L. D. Comparison of chronological and corrected ages in the gross motor assessment of low-risk preterm infants during the first year of life. Arquivos de Neuropsiquiatria. v. 64, n. 2-B, p. 418-425, 2006.

RÍOS-FLÓREZ, J. A.; NOVOA-SUAZA, A.; HERNÁNDEZHENAO, V. I. Influence of premature birth in the development of executive functions in children at a school age. Revista Chilena de Neuropsicologia. v. 12, n. 1, p. 1-11, 2017.

RYDZ, D. et al. Developmental screening. Journal of Child Neurology. v. 20, p. 4-21, 2005.

SACCANI, R.; VALENTINI, N. C. Analysis of motor development of infants from zero to 18 months of age: representativeness of the motors items of the alberta infant motor scale by age and posture. Revista Brasileira de Crescimento e Desenvolvimento Humano. v. 20, n. 3, p. 711-722, 2010.

SILVEIRA, R. C. Manual seguimento ambulatorial do prematuro de risco. Porto Alegre: Sociedade Brasileira de Pediatria, 2012.
TRONCHIN, D. M. R.; TSUNECHIRO, M. A. Prematuros de muito baixo peso: do nascimento ao primeiro ano de vida. Revista Gaúcha de Enfermagem. v. 28, n. 1, p. 79-88, 2007.

VOLPI, S. C. P. et al. Aquisição de habilidades motoras até a marcha independente em prematuros de muito baixo peso. Journal of Pediatrics. v. 86, n. 2, p. 143-148, 2010.

WORLD HEALTH ORGANIZATION. Preterm birth. Disponível em: <http://www.who.int/mediacentre/ factsheets/fs363/en/> Acesso em: 25 maio 2016.

ZANINI, P. Q. et al. Análise da aquisição do sentar, engatinhar e andar em um grupo de crianças pré-termo. Fisioterapia e Pesquisa, v. 9, n. 2, p. 57-62, 2002.

Recebido em: 2017-05-26 Aceito em: 2017-08-23 